\title{
Género, redes de amistad y rendimiento académico*
}

\section{Félix Requena Santos}

U niversidad de Santiago de Compostela. D epartamento de Sociología

15706 Santiago de Compostela. Spain

\section{Resumen}

El presente artículo examina empíricamente la relación que existe entre las redes sociales de amistad y éxito académico en base a los resultados de los exámenes, prestando especial atención a las diferencias entre hombre y mujer. El trabajo está basado en una encuesta realizada en 1992 por el D epartamento de Sociología (U niversidad de M álaga) sobre estudiantes de primer curso de la Facultad de Ciencias Económicas. En el transcurso del análisis se evidencia, por una parte, que la influencia de las relaciones de amistad sobre el resultado de los exámenes es positiva en los hombres y negativa en las mujeres; por otra parte, existe una mayor fuerza explicativa de las variables reticulares en el rendimiento de los hombres que en el de las mujeres.

Palabras clave: redes sociales, amistad, éxito académico, género.

\section{Abstract. Gender, Friendship networks and Academic success}

This paper empirically examines the relationship between friendship networks and academic success based on exam results, particulary concern to gender differences. It is based on a social survey data from Economic Faculty 1st. course undertaken in 1992 by Sociology $D$ ept. ( $M$ alaga $U$.). The main results was, on one hand, positive influence of friendship relations on exam results in men and negative in women; and the other hand, network variables have stronger incidence on men's academic success than women's.

Key words: social networks, friendship, academic success, gender.

\begin{tabular}{cc}
\multicolumn{2}{c}{ Sumario } \\
Introducción & Conclusiones \\
Datos y variables & Bibliografía \\
Análisis &
\end{tabular}

* Los datos de este artículo proceden de un proyecto de investigación para la mejora de la práctica docente en la U niversidad de M álaga, financiado por el ICE (U M A) y realizado por un equipo dirigido por el Dr. Rafael Gobernado. El autor desea agradecerle la gentileza por su consentimiento para usar dichos datos. 


\section{Introducción}

El rendimiento académico es fruto del esfuerzo y la capacidad de trabajo del estudiante. D e las horas de estudio, de la competencia y el entrenamiento para la concentración. Todo esto es verdad. Sin embargo, también hay otras muchas variables, como el entorno relacional, que inciden en el rendimiento. N uestros compañeros y amigos proporcionan el ambiente en el que una persona puede ser más o menos productiva. Así, nos interesa ver cómo afectan las variables reticulares a la productividad académica. En concreto, cómo los amigos pueden influir en el éxito académico.

Este trabajo estudia cómo y en qué proporción determinadas condiciones relacionales de los estudiantes inciden en mayor o menor medida en el resultado obtenido en el examen. Sin embargo, hay que ser conscientes de que las variables que representan situaciones relacionales, como el tamaño de la red de amigos o la densidad u homogeneidad de la red, influyen con menor fuerza en la calificación obtenida que, por ejemplo, el número de horas dedicadas al estudio de la asignatura.

La idea en la que se ha gestado este trabajo es que el apoyo social que nos proporcionan las personas con las que interactuamos es muy importante sobre determinadas condiciones de la persona. El apoyo social (H ansell, 1985) que ofrecen los que están a nuestro al rededor suministra una gran protección, seguridad, bienestar, etc., al mismo tiempo que proporcionaría la fuerza y la confianza suficiente para superar el estrés y los inconvenientes que sufren los alumnos ante los temidos exámenes.

Las redes sociales vinculan personas de igual o diferentes estatus en la estructura social, tanto directa como indirectamente. $D$ entro de ellas circula tanto información como bienes y servicios, o apoyo material o emocional. Principalmente se basan en este hecho, pero también pueden proporcionar presiones, roces y, sobre todo, control social (Leffler, Krannich y Gillespie, 1986; Requena, 1989). El propósito de esta investigación es conocer empíricamente qué relación hay entre las redes de amistad y el éxito en los exámenes, en ambos sexos en general y entre los diferentes géneros en particular, pues entendemos que las actitudes relacional es varían de hombre a mujer (W instead, 1986). La idea es la de realizar un estudio exploratorio con objeto de aumentar el acopio de estudios basados en el concepto de red social, dado el escaso número de estos trabajos que existen sobre la realidad social española.

En este trabajo se usa una pequeña variación sobre el concepto de red social. Se trata de la red personal o red centrada en el respondente. Esta red consiste en todos los vínculos que llegan o parten directamente del sujeto (Requena, 1996). Esta red describe el entorno social inmediato del actor. Es decir, es la red centrada exclusivamente en una persona: el encuestado. En este caso, se presta atención exclusivamente a los vínculos de amistad. D eberíamos Ilamarlas «redes personales de amistad». Por comodidad las Ilamaremos, simplemente, «redes de amistad». N o se trata, por tanto, de la red total del sujeto.

$\mathrm{N}$ os apoyaremos en las relaciones causal es entre las relaciones de amistad 
y el rendimiento académico, para probar que las relaciones personales de amistad afectan al resultado académico. Las relaciones entre el rendimiento acadé mico y las relaciones de amistad se pueden explicar a través de las siguientes proposiciones:

1. D e acuerdo con ciertas directrices de la sociología de la educación (Bidwell y Friedkin, 1988), el éxito o el fracaso escolar dependen entre otros factores de la tolerancia educativa. En otras palabras, de la capacidad del alumno para aguantar las incomodidades y presiones provenientes de la escolarización. D e este modo, cuanto mayor sea esta tolerancia del alumno, menores serán los costes, emocionales entre ellos, de seguir con los estudiosy, por tanto, mayores los beneficios.

2. Los alumnos pueden adoptar dos actitudes frente al profesor: los que se identifican con él. Son alumnos que se hacen responsables y trabajadores. 0 tros, por el contrario, se identifican con sus iguales, con los amigos, y tra tan de romper la disciplina escolar. El que un alumno adopte una u otra identificación no tienen nada que ver con su capacidad intelectual. Sin embargo, este posicionamiento constituye el origen del éxito 0 del fracaso escolar futuro (Gobernado, 1991)1. Apoyándonos en esta proposición, podemos matizar la hipótesis inicial: las relaciones de amistad sirven de apoyo eficaz al rendimiento educativo dentro de ciertos límites. Esto nos lleva a suponer que los niveles intermedios (ni altos, ni bajos) de tamaño, densidad y homogeneidad de las redes serán más efectivos para la integración del estudiante en su entorno, lo que repercutirá en su rendimiento. Cuando las relaciones de amistad exceden tales límites, la consecuencia es que el alumno se integra en un grupo de iguales, el cual por definición está en conflicto con la jerarquía desigualitaria de la organización educativa.

3. H asta qué punto las relaciones de amistad constituyen un apoyo para el alumno. Las redes de amigos constituirían un apoyo que aumentaría la tolerancia educativa del alumno y, por lo tanto, incrementaría las probabilidades de éxito académico.

Asimismo, de acuerdo con otras investigaciones (M oore, 1990; Requena, 1994), existen diferencias significativas entre la composición de las redes personales de los hombres y de las mujeres. Además nosotros intuimos al rea-

1. Verdaderamente la proposición indicada puede general izarse a cual quier organización formal. Así, toda organización formal alberga en su seno una fuente constante de discordia. Los individuos que desempeñan un mismo rol pueden adoptar una doble actitud: aceptar la jerarquía social (de roles) y orientarse al desempeño eficaz de su tarea o, por el contrario, identificarse con sus iguales (del mismo rol) y rechazar el orden organizativo existente. El resultado es la presencia constante de dos colectivos opuestos y en conflicto dentro de la categoría definida por desempeñar un mismo rol: por un lado los «responsables» y trabajadores, por otro los «díscolos» y saboteadores de la organización establecida. También puede verse T. Parsons (1976). 
lizar las entrevistas que estas diferencias no sólo se daban en la composición de las redes, sino también en el funcionamiento de las redes de amistad según cual sea el sexo.

4. ¿Tienen iguales propiedades respecto al éxito en los exámenes las redes de amigos de los hombres que las de las mujeres? Trataremos de analizar las diferencias reticulares existentes entre las redes según los géneros.

\section{D atos y variables}

Los datos de este estudio se han obtenido mediante dos encuestas Ilevadas a cabo por el D epartamento de Sociología de la U niversidad de M álaga. La primera durante el mes de noviembre de 1991, tenía por objeto conseguir información de la muestra y obtener todos los datos socio-demográficos. La segunda, compuesta por tres cuestionarios, durante abril y mayo de 1992 para obtener información sobre las relaciones de amistad y sobre la calificación del examen, así como de las horas dedicadas tanto al estudio como a salir de casa. El tamaño de la muestra fue $\mathrm{N}=404$ y se realizó entre jóvenes de ambos sexos y estudiantes de primer curso del turno diurno de la Facultad de Ciencias Económicas y Empresariales de la U niversidad de M álaga. Con objeto de satisfacer los requisitos de nuestro trabajo, se seleccionó una muestra muy homogénea. D e esta forma, del total de la muestra se escogieron aquellos estudiantes que cumplían las siguientes características: edad 17-18 años; eran alumnos que no habían repetido ningún curso antes de llegar a la universidad; no eran repetidores ni venían de otra licenciatura; vivían en casa con su familia de origen. Los que cumplían estos requisitos eran $\mathrm{N}=137$, con lo que la muestra original se vio afectada por una mortalidad del 33,9\%.

Todos los alumnos fueron interrogados acerca de sus «amigos». Los cuestionarios se administraron en clase y su cumplimentación fue dirigida por alguno de los miembros del equipo investigador. No se realizó una definición previa del concepto de amistad, con objeto de no condicionar la concepción de amigo que tuviera cada uno de los respondentes. $\mathrm{N}$ os basamos en la idea de que la «amistad» no puede ser definida unívocamente, sino que lo único que todos los individuos reconocen es el término (el sonido o la grafía) amistad. Esto permite que cada cual piense lo que quiera sobre tal término (es decir, el significado es muy personal) y al mismo tiempo haya acuerdo sobre el mismo (el significante es lo completamente colectivo). Sin duda, la definición del concepto de amistad resulta un problema. Ante esta situación los investigadores han tratado de solventarlo de tres formas diferentes: primera, la que deja a la responsabilidad de los sujetos de estudio el que ellos mismos definan lo que entienden por amigo; segunda, la que limita la definición de amigo de alguna forma, y tercera, la que emplea métodos inductivos capaces de determinar lo que por término medio significa la amistad (Requena, 1994). Si consideramos que el significado es lo personal y el significante es lo colectivo, entonces, el primer tipo de solución al problema es la menos mala de las tres. Ésta es la que hemos escogido. 
Las variables examinadas se midieron de la siguiente forma:

a) Resultado del examen: es la calificación obtenida en el primer parcial de sociología de los al umnos antes mencionados. Se trata de una variable continua. Cuando se recodifica para que tome una forma cual itativa toma los siguientes valores:

$1=$ no presentado;

2 = de 0 hasta 4 , suspenso, y

$3=$ desde 5 hasta 10, aprueban.

Los resultados generales fueron que el $20 \%$ de los estudiantes no se presentaron; el 45,2\% resultó suspenso, y el 34,8\% aprobó el examen.

b) N umero de ami gos o tamaño dela red: es el número total de amigos nombrado por cada respondente. $\mathrm{N}$ osotros lo identificamos con el tamaño de la red de amigos del sujeto. Es necesario matizar que se trataban de amigos compañeros de clase. El objetivo era saber si estudiaban juntos y si ese apoyo afecta al éxito académico.

c) D ensidad de la red: es el grado de conexión interna de la red, es decir, la proporción existente entre los vínculos reales entre los individuos de la red y todos los vínculos posibles. Se calcula mediante la fórmula: $D=200 \mathrm{a} / \mathrm{n}(\mathrm{n}-1)=\%$; donde a es el número de vínculos que existen y $\mathrm{n}$ es el número de individuos que componen la red (Requena, 1989). Así, por ejemplo, un sujeto que nombre a tres amigos y a su vez uno de ellos conoce a los otros dos pero estos dos últimos no se conocen entre sí tendría una densidad del 83,3\%. Para saber cuantos vínculos establece cada estudiante, se les entregó una hoja con una estrella en la que aparecían siete recuadros (número máximo de amigos permitidos). A continuación ponían el nombre del respondente y el de sus amigos trazando líneas entre los nombres correspondientes en cada recuadro y el nombre del encuestado.

d) H omogeneidad de la red: se entiende por homogeneidad de la red el grado de igualdad interna de la misma, en otras palabras, el grado en el que los componentes de tal red poseen algún rasgo común. Una red es muy homogénea cuando todos los miembros son iguales con respecto a al gún rasgo. Una red es poco homogénea cuando cada miembro de la red posee rasgos distintos los unos de los otros. El cálculo se efectuó siguiendo estas directrices: la homogeneidad consiste en la razón existente entre la suma de las distancias entre los valores de la variable considerada en cada red de amigos y el total de amigos. Entre dos redes de igual homogeneidad pero de diferente tamaño, es más homogénea la de tamaño mayor. La homogeneidad de una red de amigos puede ser observada con respecto a múltiples dimensiones: Así puede haber homogeneidad con respecto a la edad, cuando todos tengan la misma; con respecto al sexo, si todos son hombres o todas son mujeres; con respecto a la educación; la actividad; la clase social; etc. N osotros establecimos cuatro matices de homogeneidad:

- H omogeneidad en la edad: hay homogeneidad máxima en edad cuan- 
do todos los entrevistados tienen la misma edad, independientemente de que sean más o menos jóvenes.

- Homogeneidad en el sexo: máxima cuando son del mismo sexo, independientemente del sexo de que se trate.

- Homogeneidad en la educación: máxima cuando todos tienen el mismo nivel de educación.

- Homogeneidad en la duración de la amistad: máxima cuando todos los miembros de la red se conocieron en la misma época, con independencia de que haga mucho o poco tiempo de tal acontecimiento.

e) $\mathrm{H}$ oras de estudio el martes por la tarde.

f) H oras de estudio el sábado por la tarde.

g) H orasfuera de casa el martes por la tarde.

h) H oras fuera de casa el sábado por la tarde: estas cuatro variables, ya se trate de horas de estudio o de salida de casa, hacen referencia al martes o al sábado anterior al día en que se realizó la entrevista.

\section{Análisis}

En la tabla 1 se pueden apreciar las diferencias entre todas las variables consideradas comparando a los hombres con las mujeres. La principal diferencia entre los sexos, atendiendo a la significación de la diferencia entre ambos grupos, es la homogeneidad respecto a la duración de la amistad y las horas que pasan junto con los amigos en los días entre semana, siendo en los dos casos superior en los hombres.

Respecto a las variables reticulares, como la densidad o el tamaño de la red, hay diferencias aunque no son significativas. Los hombres jóvenes tienen redes de amistad más grandes. Sin embargo, las mujeres jóvenes presentan una mayor densidad en las redes de amigos. La diferencia es pequeña, pero sin duda hay que tenerla en consideración.

Tabla 1. D iferencias de las variables por sexo.

\begin{tabular}{llll}
\hline & $\begin{array}{l}\text { H ombres } \\
\text { media } \pm \text { s.d. }\end{array}$ & $\begin{array}{l}\text { Mujeres } \\
\text { media } \pm \text { s.d. }\end{array}$ & $\begin{array}{l}\text { Significación } \\
\mathbf{p}^{*}\end{array}$ \\
\cline { 2 - 4 } Result. exam. & $2,33 \pm 2,02$ & $2,70 \pm 2,01$ & $<0,05$ \\
Tamaño de red & $6,29 \pm 1,85$ & $5,93 \pm 1,98$ & n.S. \\
D ensidad de red & $69,71 \pm 23,17$ & $70,85 \pm 23,65$ & n.S. \\
H om. de edad & $0,98 \pm 1,04$ & $0,79 \pm 1,05$ & n.S. \\
H om. de sexo & $0,73 \pm 0,78$ & $0,59 \pm 0,67$ & n.S. \\
H om. de educación & $2,36 \pm 2,5$ & $1,69 \pm 2$ & n.S. \\
H om. duración de la amist. & $10,55 \pm 7,5$ & $7,51 \pm 5,18$ & $<0,01$ \\
H oras estudio martes** & $4: 32 \pm 2: 15$ & $4: 33 \pm 2: 02$ & n.S. \\
H oras estudio sábado & $2: 34 \pm 2: 38$ & $2: 54 \pm 2: 43$ & n.S. \\
H oras fuera martes & $2: 39 \pm 2: 01$ & $1: 33 \pm 2: 34$ & $<0,05$ \\
H oras fuera sábado & $4: 26 \pm 3: 14$ & $4: 18 \pm 3: 19$ & n.S.
\end{tabular}

${ }^{*} \mathrm{H}$ ombres respecto a mujeres; ${ }^{* *} \mathrm{H}$ oras y minutos; n.s. $=$ no tiene significación estadística. 
Respecto a otras variables que inciden más directamente sobre el resultado académico (por ejemplo, horas de estudio del sábado), son mayores en las mujeres que en los hombres. Aunque según se aprecia en la tabla 1, las horas de estudio del sábado puede indicar que se trata de alumnos a los que no les rinde demasiado el estudio durante la semana (martes) y tienen, de alguna forma, que recuperar en lugar de salir a divertirse.

En las tablas 2 y 3 se muestran las correlaciones entre todas las variables consideradas y su significación según el género y la población total respectivamente. En ellas se aprecia que al gunas variables reticulares actúan de forma

Tabla 2. Correlaciones entre todas las variables (hombres y mujeres)*.

$\mathbf{A}=\mathrm{N}$ úmero de amigos (tamaño de la red); $\mathbf{B}=$ Resultado del examen; $\mathbf{C}=\mathrm{H}$ oras de estudio del martes; $\mathbf{D}=\mathrm{H}$ oras de estudio del sábado; $\mathbf{E}=\mathrm{H}$ oras fuera de casa el martes; $\mathbf{F}=\mathrm{H}$ oras fuera de casa el sábado; $\mathbf{G}=\mathrm{D}$ ensidad de la red; $\mathbf{H}=\mathbf{H}$ omogeneidad de edad; I = H om. de sexo; J = H om. de educación; $\mathbf{K}=$ = om. de tiempo de amistad.

\begin{tabular}{lcccccccccccc}
\hline & $\mathbf{A}$ & $\mathbf{B}$ & $\mathbf{C}$ & $\mathbf{D}$ & $\mathbf{E}$ & $\mathbf{F}$ & $\mathbf{G}$ & $\mathbf{H}$ & $\mathbf{I}$ & $\mathbf{J}$ & $\mathbf{K}$ \\
\cline { 2 - 11 } $\mathbf{A}$ & - & 0,10 & 0,26 & $-0,04$ & 0,12 & 0,09 & $-0,16$ & $0,47^{\mathrm{b}}$ & $0,48^{\mathrm{b}}$ & $0,39 \mathrm{a}$ & $0,57^{\mathrm{b}}$ \\
$\mathbf{B}$ & $-0,02$ & - & 0,05 & 0,09 & 0,18 & $-0,23$ & $-0,07$ & $-0,15$ & 0,05 & 0,02 & 0,23 \\
$\mathbf{C}$ & $0,23 \mathrm{a}$ & $-0,10$ & - & $0,47 \mathrm{~b}$ & 0,04 & $-0,16$ & 0,03 & 0,02 & $-0,08$ & 0,34 & 0,08 \\
$\mathbf{D}$ & 0,11 & $-0,06$ & 0,19 & - & 0,28 & $-0,54 \mathrm{~b}$ & 0,32 & $-0,22$ & $-0,03$ & 0,03 & $-0,14$ \\
$\mathbf{E}$ & $-0,07$ & 0,14 & $-0,40^{\mathrm{b}}$ & $-0,24$ & - & $-0,03$ & $-0,06$ & $-0,11$ & 0,02 & 0,05 & $-0,12$ \\
$\mathbf{F}$ & 0,01 & 0,09 & $-0,12$ & $-0,59 \mathrm{~b}$ & 0,19 & - & $-0,09$ & 0,03 & $-0,01$ & 0,04 & 0,01 \\
$\mathbf{G}$ & $-0,38 \mathrm{~b}$ & 0,05 & $-0,23$ & $-0,05$ & 0,01 & 0,12 & - & $-0,34$ & 0,13 & $-0,21$ & $-0,18$ \\
$\mathbf{H}$ & $0,39 \mathrm{~b}$ & $-0,04$ & 0,13 & 0,18 & - & $-0,10$ & $-0,22$ & - & 0,32 & $0,52^{\mathrm{b}}$ & $0,53^{\mathrm{b}}$ \\
$\mathbf{I}$ & $0,41^{\mathrm{b}}$ & $-0,12$ & 0,08 & 0,24 & 0,05 & $-0,20$ & $-0,20$ & 0,15 & - & 0,16 & $0,44^{\mathrm{b}}$ \\
$\mathbf{J}$ & $0,34 \mathrm{~b}$ & 0,14 & 0,23 & 0,22 & $-0,03$ & $-0,09$ & $-0,14$ & $0,51^{\mathrm{b}}$ & 0,14 & - & $0,45^{\mathrm{b}}$ \\
$\mathbf{K}$ & $0,29 \mathrm{a}$ & 0,12 & 0,08 & 0,06 & $-0,11$ & 0,06 & $-0,16$ & 0,14 & 0,01 & 0,23 & - \\
\hline
\end{tabular}

${ }^{*} \mathrm{H}$ ombres $(\mathrm{N}=48)$ por encima de la diagonal; mujeres $(\mathrm{N}=83)$ por debajo de la diagonal. a) $p<0,01$; b) $p<0,001$.

Tabla 3. Correlaciones entre todas las variables (población total).

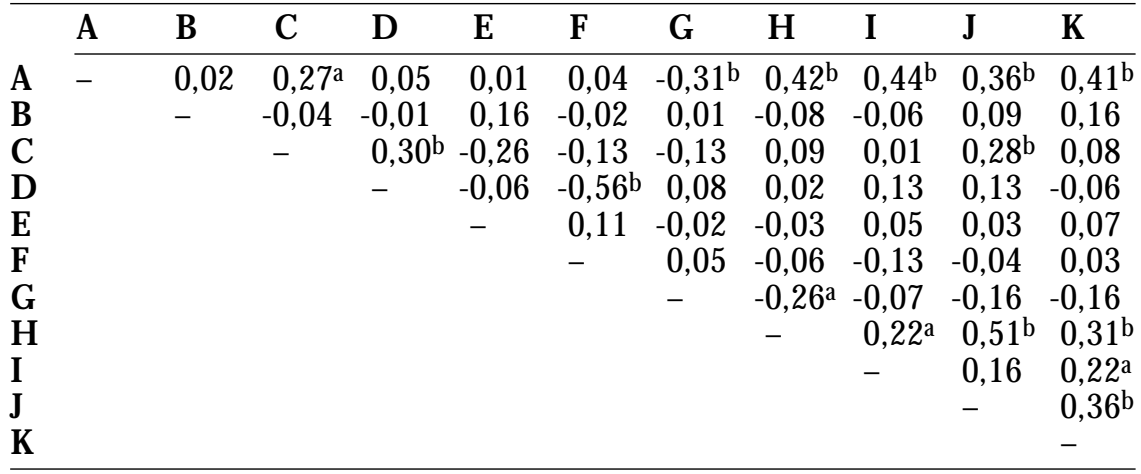

a) $p<0,01 ;$ b) $p<0,001$. 
diferente en los hombres que en las mujeres. El tamaño de la red tiene una relación positiva con el resultado del examen en los hombres, mientras que la relación es negativa en el caso de las mujeres.

El análisis de regresión múltiple (tabla 4) muestra la diferencia que se produce en la fuerza explicativa de la variación de las varianzas de las variables estudiadas sobre el rendimiento académico en función de los sexos $\left(R^{2}=26 \%\right.$ para los hombres y $R^{2}=9,41 \%$ para las mujeres). Existe un gran parecido entre la proporción de varianza explicada en el análisis de regresión de la población total y la de las mujeres ( $R^{2}=9,47 \%$ y $R^{2}=9,41 \%$ respectivamente).

Estos datos muestran que la primera y la tercera proposiciones se cumplen más en los hombres que en las mujeres. En los hombres la relación entre tamaño de la red y rendimiento académico es positiva mientras que en las mujeres es negativa (tabla 2). Además, en lo que se refiere a la primera proposición,

Tabla 4. C oeficientes de regresión múltiple estandarizados para los hombres y las mujeres.

\begin{tabular}{llcc}
\hline Variables independientes & \multicolumn{3}{c}{ Variable dependiente: resultado del examen } \\
\hline & H ombres & M ujeres & Todos \\
\cline { 2 - 4 } N úm. amigos (tamaño de red) & 0,014 & 0,033 & 0,048 \\
H oras estudio martes & $0,062^{*}$ & $-0,076$ & $-0,049$ \\
H oras estudio sábado & $-0,165$ & $-0,003^{*}$ & $-0,022^{*}$ \\
H oras fuera sábado & $-0,298$ & 0,133 & $-0,088^{*}$ \\
H oras fuera martes & 0,215 & $0,015^{*}$ & 0,160 \\
D ensidad de la red & $-0,096$ & $0,028^{*}$ & $0,02^{*}$ \\
H omogeneidad edad & $-0,419$ & $-0,139$ & $-0,187$ \\
H omogeneidad sexo & $*$ & $-0,139$ & $-0,116$ \\
H omogeneidad educación & $0,004^{*}$ & 0,219 & 0,125 \\
H omogeneidad tiempo amistad & 0,421 & 0,105 & 0,201 \\
R2 & $26 \%$ & $9,41 \%$ & $9,47 \%$ \\
\hline
\end{tabular}

*no tiene significación estadística

Tabla 5. Características de la red y rendimiento académico.

\section{Variables independientes}

Tamaño de red Densidad de red H omogeneidad edad H omogeneidad sexo H omogeneidad duración de la amistad

\section{Variable dependiente: resultado del examen}

Aprueban Suspenden No presentados Significación

\begin{tabular}{llll}
\hline$\uparrow \downarrow$ & $\uparrow$ & $\downarrow$ & n.s. \\
$\uparrow \downarrow$ & $\downarrow$ & $\uparrow$ & n.s. \\
$\uparrow$ & $\uparrow \downarrow$ & $\uparrow \downarrow$ & $*$ \\
$\uparrow \downarrow$ & $\downarrow$ & $\uparrow \downarrow$ & n.s. \\
$\downarrow$ & $\uparrow \downarrow$ & $\uparrow$ & $* *$
\end{tabular}

$* p<0,01 ; * * p<0,05 ; n .5 .=$ no tiene significación estadística.

$\uparrow$ muy alta; $\downarrow$ muy baja; $\uparrow \downarrow$ nivel intermedio. 
ésta se confirma con el hecho de que en el caso de los varones, el coeficiente con más peso respecto al resultado del examen sea la homogeneidad en el tiempo de duración de la amistad (tabla 4). Es evidente que el éxito/fracaso escolar está muy influenciado por la duración de la amistad, hecho que afecta a la tolerancia educativa. Asimismo, se muestra también la diferencia como consecuencia del género, como se preveía en la cuarta proposición.

Por último, la segunda proposición parece confirmada a la luz de la tabla 5 , donde se aprecia que los extremos muchas veces tienen comportamientos parecidos. Tanto los que tienen redes muy grandes como los que se mueven dentro de redes pequeñas, los resultados académicos no son muy buenos, suspenden o no se presentan respectivamente. Los que aprueban tienen redes de tamaño intermedio. Lo mismo ocurre con la densidad de la red: sólo aprueban aquellos que tienen densidad intermedia.

\section{Conclusiones}

A la vista de los datos podemos destacar los siguientes puntos relevantes. Ante todo, existe relación estadística entre los resultados del examen y el resto de las variables, aunque con una significación estadística muy escasa. Estas relaciones ponen de manifiesto los siguientes hechos:

1. Existe una gran diferencia entre los hombres y las mujeres en lo que respecta a las relaciones entre las variables reticulares y el rendimiento académico.

2. La fuerza explicativa de las variables reticulares sobre el rendimiento académico es tres veces mayor en los hombres que en las mujeres; lo mismo ocurre en los hombres con respecto a la población total.

3. A juzgar por los datos, los resultados que facilitan los extremos (mucho 0 poco) de los rasgos de la red tienen comportamientos parecidos, y sólo los niveles intermedios actúan de forma diferente.

Estos resultados evidencian, como era de esperar, que el peso de las relaciones de amistad entre estudiantes universitarios sobre su rendimiento académico no puede ser muy grande, salvo en casos particulares y desechables en los grandes números. Además, los rasgos de la red (tamaño, densidad y homogeneidad) se caracterizan por su abstracción y relativa lejanía de la actividad estudiada (el rendimiento académico).

Las relaciones entre el tamaño de la red y el rendimiento académico carecen de significación estadística. D e cualquier forma, los datos indican que los niveles intermedios son más eficaces que los extremos, en lo que a rendimiento académico se refiere, lo que avala la hipótesis de partida.

Algo parecido ocurre con la densidad de la red. D entro de unos niveles de significación algo superiores, también aparentan más eficacia los niveles intermedios (más aprobados). Sin embargo, el análisis de la densidad requiere mayor atención ya que plantea problemas complejos: en general, la densidad 
alta proporciona al individuo seguridad en sí mismo, certeza en las ideas y opiniones; por el contrario, la densidad baja favorece la inseguridad, la duda y el aislamiento. Esto puede constituir un arma de doble filo a la hora de contribuir al éxito 0 al fracaso académico. Una densidad y homogeneidad altas proporcionarían escasa información, mientras que densidades y homogeneidades bajas son fuente abundante de información. De cualquier forma, la bipolaridad anterior apunta más claramente hacia los que tienen una red con baja densidad.

D e todos los indicadores de homogeneidad el que más interés tiene en nuestro trabajo es el de la edad. Según los datos es más eficaz para el objetivo académico una red homogénea en edad. Sin embargo, la homogeneidad en lo concerniente al sexo no es oportuna, siempre según los datos. Por su parte, es difícil interpretar los resultados sobre homogeneidad con respecto a la educación: es más eficaz una homogeneidad mínima, tanto en lo referente a aprobar cuanto en lo referente a estudiar.

\section{Bibliografía}

Bidwell, Ch.E.; FrIed KIN , N .E. (1988). «T he Sociology of Education». En SM ELSER, N. H andbook of Sociology. Londres: Sage.

Go BERNAD 0, R. (1991). «La quiebra de las unanimidades teóricas: las relaciones de roles». Revista Española de Investigaciones Sociológicas, núm. 54, p. 51-64.

H AN SELL, S. (1985). «Adolescent friendship networks and distress in scholl». Social Forces, núm. 63, p. 698-715.

Leffler, A.; KRAN N ICH, R.S.; GILlesPIE, D.L. (1986). «C ontact, support, and friction». Sociological Perspectives, núm. 29, p. 337-455.

M O ORE, G. (1990). «Structural determinants of men's and women's personal networks». American Sociological Review, núm. 55, p. 726-735.

PARSO N S, T. (1976). «La clase como sistema social: al gunas de sus funciones en la sociedad americana». En G RAS, A. Textos fundamentales de Sociología dela Educación. $M$ adrid: N arcea, p. 53-60.

REQUENA, F. (1989). «EI concepto de red social». Revista Española de Investigaciones Sociológicas, núm. 48, p. 137-152.

- (1994). Ami gos y redes sociales. M adrid: CIS/Siglo XXI.

- (1996). Redes sociales y Cuestionarios. M adrid: CIS.

W IN STEAD, B.A. (1986). «Sex differences in samesex friendships». En D ERLEGA, V.J.; W IN ST EAD, B.A. (ed.). Friendships and social Interaction. N ueva York: SpringerVerlag, p. 81-99. 